\title{
Novel quantum phases and mesoscopic physics in quantum gases
}

\author{
F. Chevy, R. Citro, G. Lamporesi, A. Minguzzi, A. Recati, and T. Roscilde
}

This volume of The European Physical Journal Special Topics is a collection of original papers presented either as oral contributions or as posters at the Seventh International Workshop "Quantum Gases and Quantum Coherence" held in Trento (Italy) from the $28^{\text {th }}$ to the $31^{\text {st }}$ of May 2014. This event has been organized with five sessions (Matter Waves, Strongly Correlated Atomic Systems, Artificial Gauges in Atomic Systems, Quantum Simulators, Dipolar Gases) each opened by an overview lecture given by a senior researcher, expert in the fields of quantum gases and condensed matter (theory and experiments) and has involved mainly students and young researchers both as speakers and participants.

In the last two decades, ultracold quantum gases have become a very versatile testing ground for quantum many-body theories due the amazing experimental feasibility which allows changing the dimensionality of the system, introduce disorder in a controlled way, create a whole variety of strongly correlated states and introduce synthetic gauge fields. This possibility, along with the opportunity of simulating Hamiltonians, has rendered the connection between the physics of ultracold quantum gases and the physics at mesoscopic scale very solid. This connection is clearly evident in the selection of contributions for the conference. The papers collected in this topical issue represent a cross section of the oral and poster contributions in the Workshop and contain original results on five relevant research directions in the field of quantum gases.

The topics covered by the volume are:

Matter waves - Lecturer Alice Sinatra

A specific feature of ultra cold atomic gases in optical or magnetic potentials, is that they are well isolated from the environment. Added to tunable interactions, this makes the appealing possibility to generate entangled states for quantum metrology. In a bimodal Bose-Einstein condensate, useful quantum correlations in the form of spin-squeezing result from the interaction-induced phase dynamics at short times. In this frame, theoretical results have been presented about the fundamental limits of spin-squeezing in condensates due to finite temperature and decoherence.

Another important topic is that of Anderson localization. In the absence of interaction, it is well known that one and two dimensional systems have only localized states in the presence of disorder, while in three dimensions for weak disorder high energy extended states and low energy localized states are separated by a mobility edge, and for strong disorder all states are localized. In the absence of interaction, the main focus is on correlated and anisotropic disorder and the resulting metal insulator transition is well understood thanks to analytical and numerical work. The 
observation of Anderson localization in 2008 has led to a considerable activity in the field of disorder and cold atoms.

Strongly correlated atomic systems - Lecturer Immanuel Bloch

Another important topic is that of strong correlation physics with ultracold atoms in optical lattices. Ultracold atoms in optical lattice form an ideal test bed to realize and explore strongly correlated quantum many-body systems. In particular recent highresolution imaging and control techniques probe and control quantum many-body systems in an unprecedented way. Among the touched topics there are examples regarding quantum magnetism: the direct detection of a more than 80 year old prediction by Hans Bethe on bound-magnon states in the Heisenberg model, the observation of the propagation of single spin impurities in the Heisenberg model and the direct measurement of entanglement generation during their propagation and the realization of novel long-range interacting quantum spin models using Rydberg atoms.

Artificial gauges in atomic systems - Lecturer Nigel Cooper

Atomic quantum gases are neutral, and therefore, not affected by external electromagnetic fields in the way electrons are. This constitutes a central issue towards the quantum simulation of solid state models involving an external magnetic field, e.g. the Quantum Hall Effect. In the presence of complex hopping parameters on a lattice, the atomic gas can mimic the dynamics of an electron gas subjected to an external magnetic field. Indeed an effective Aharonov-Bohm phase can be engineered if the atomic wave function acquires a nontrivial phase while tunneling around a single plaquette of the lattice. This has recently opened a new research line in the cold atoms context which concerns the implementation of uniform artificial magnetic fields in optical lattices and observation of a Meissner-like to vortex phase transition with bosonic atoms in ladders or the realization of spin-orbit-coupled quantum gases with Rashba and Dresselhaus spin-orbit coupling.

Quantum simulators - Lecturer Ferdinand Schmidt-Kaler

Among the systems that have been recently proposed as quantum simulators fall the ions confined in a Paul trap arranged in linear crystals, the spin-boson models and the fermions with tunable spin. As for the trapped ions, segmented traps provide the promising architecture for a future quantum computer, supposed that transport and the splitting of ion crytsals can be performed faster than quantum logic gate operations while the ion's motional state still allows for high-quality gates. Hybrid lattice models with interacting spin and bosons give instead the possibility of deriving fundamental limits for the speed of propagation of spin-spin correlations (i.e. Lieb-Robinson bounds) and the existence of a quantum phase transition in the Ising universality class that involves spins and boson on the same footing. Liquids of Fermions with tunable spin, instead represents a new playground to realize systems beyond the spin $1 / 2$ Luttinger model. Degenerate 173Y F Fermi gases in 1D are characterized by a large nuclear spin and highly-symmetric atom-atom interactions in the ground level manifold, which result in the possibility of performing quantum simulation of systems with intrinsic $\mathrm{SU}(\mathrm{N})$ symmetry with high $\mathrm{N}$ that have no analogous in solid state systems.

Dipolar gases - Lecturer Tilman Pfau

The realization and the control of long-range interactions in atomic systems at very low temperatures opens up a whole new realm of many-body physics that has recently become a central focus of research. In this respect Rydberg gases are very well-suited to achieve this goal, as the van der Waals forces between them are many orders of magnitude larger than for ground state atoms. When the electronic ground state is off-resonantly coupled to a highly excited state with strong binary interactions, the 
two body interaction is modified into a soft core potential. Importantly, despite the strong repulsion between the admixed Rydberg states, the dressing of the ground state does not lead to atomic trap-loss, both in free space and in optical lattices. At the many body level these non-local interactions can provide an optimal playground for the engineering of exotic many body phases. The ability to control and tune interactions and particle numbers in such systems allows the creation of superfluids, crystalline states as well as the long sought supersolid phase.

All the papers have been peer reviewed following the usual standards of The European Journal of Physics Special Topics. We would like to thank the Chief Editors for giving us the possibility of publishing this topical issue, and all the staff in the persons of Agnès Henri, Sabine Lehr, Isabelle Houlbert.

This Workshop was made possible thanks to the financial support from INO-CNR BEC Center in Trento, Provincia Autonoma di Trento, the Physics Department of Trento through the ERC grant QGBE, the Physics Department "Caianiello" of Salerno, Laser \& Electro-Optic Solutions, Cassa Rurale di Levico Terme and Comune di Levico. The editors also acknowledge the precious collaboration of Rachele Zanchetta in the contacts process for this volume. 